\title{
WATER ICE ON THE SATELLITE OF KUIPER BELT OBJECT 2003 EL $_{61}$
}

\author{
K. M Barkume, M. E. Brown, and E. L. Schaller \\ Division of Geological and Planetary Sciences, California Institute of Technology, Pasadena, CA 91125; \\ barkume@caltech.edu,mbrown@caltech.edu, schaller@caltech.edu \\ Received 2006 January 24; accepted 2006 February 3; published 2006 February 28
}

\begin{abstract}
We have obtained a near-infrared spectrum of the brightest satellite of the large Kuiper Belt object $2003 \mathrm{EL}_{61}$. The spectrum has absorption features at 1.5 and $2.0 \mu \mathrm{m}$, indicating that water ice is present on the surface. We find that the satellite's absorption lines are much deeper than water ice features typically found on Kuiper Belt objects. We argue that the unusual spectrum indicates that the satellite was likely formed by impact and not by capture.
\end{abstract}

Subject headings: comets: general — infrared: solar system — minor planets, asteroids

\section{INTRODUCTION}

Satellites in the Kuiper Belt provide us with unique insights into the internal properties of icy bodies as well as the early dynamical history of the trans-Neptunian region. To date, over a dozen satellites have been found around Kuiper Belt objects (KBOs), and the overall satellite fraction is estimated to be $11_{-2}^{+5} \%$ (Stephens \& Noll 2006). The first KBO satellites discovered (excluding the Pluto-Charon system) were found to have high eccentricities, large orbital separations, and similar brightness to the primary, indicating that the satellites are nearly as large as their parent. Various capture mechanisms were proposed to account for the high specific angular momentum of these systems (Goldreich et al. 2002). Recently, Brown et al. (2006) have found several satellites around the largest known KBOs, and these satellites appear to be different from those discussed above. The satellite fraction appears to be higher around large KBOs, and like the Pluto-Charon system, the satellites have lower fractional brightness compared to the primary. The orbit of the brightest satellite around $2003 \mathrm{EL}_{61}$ is nearly circular, and Brown et al. (2006) suggest that these systems likely formed by a different mechanism and may be the result of giant impacts (McKinnon 1989; Canup 2005). The density, albedo, and surface composition of these satellite systems may help us to clarify the origins of satellites in the Kuiper Belt.

Near-infrared spectroscopy is a particularly well-suited method for remotely studying the surface composition of KBOs. Many of the abundant species in the outer solar system, including $\mathrm{CH}_{4}$, $\mathrm{CO}_{2}$, and $\mathrm{H}_{2} \mathrm{O}$ ices, have absorption features in the near-IR. Pluto and other large KBOs have spectra that are dominated by $\mathrm{CH}_{4}$ (Cruikshank et al. 1976; Brown et al. 2005; Licandro et al. 2006). A small amount of water ice has been observed on a number of KBOs, and some show evidence for the presence of other ices (Fornasier et al. 2004 and references therein). Charon, the only other KBO satellite for which a spectrum has been published, has strong features due to crystalline water ice absorptions (Brown \& Calvin 2000). The spectra of satellites may shed light on their origin. Specifically, captured objects are likely to have spectra similar to other KBOs, but satellites formed from other mechanisms could show unusual or altered surface compositions.

$2003 \mathrm{EL}_{61}$ is currently the third brightest known KBO and is elliptical in shape, with axes of 1960 and $2500 \mathrm{~km}$ (Rabinowitz et al. 2006). The near-IR spectrum of $2003 \mathrm{EL}_{61}$ is similar to Charon's and shows strong water ice absorption features (Trujillo et al. 2005). Its satellites offer us an excellent oppor- tunity to study the formation and dynamics of KBO binaries. The brighter satellite, S/2005 (2003 $\left.\mathrm{EL}_{61}\right)$ 1, has a period $49.12 \pm 0.03$ days and a maximum separation of 1".4 (Brown et al. 2005). The orbit is nearly circular, with an eccentricity of 0.05. The inner satellite, $\mathrm{S} / 2005$ (2003 $\left.\mathrm{EL}_{61}\right)$ 2, appears to have an orbit of $34.7 \pm 0.1$ days, although there are currently insufficient data to reliably fit a noncircular orbit. The inner satellite has a fractional brightness of $1.5 \%$ compared to 2003 $\mathrm{EL}_{61}$, making it difficult to study with spectroscopic techniques. The outer, brighter satellite has a fractional brightness of 5\% and is a viable target for low-resolution infrared spectroscopy. We report here on the near-IR ( $J$ - through $K$-band) spectrum of $2003 \mathrm{EL}_{61}$ 's brighter satellite. We compare the spectrum of the outer satellite to $2003 \mathrm{EL}_{61}$ 's as well as to other KBOs. We discuss possible formation scenarios for the satellite and find that the surface composition is consistent with formation by giant impact.

\section{OBSERVATIONS}

Observations of $2003 \mathrm{EL}_{61}$ were obtained on the Keck Telescopes on UT 2005 April 26 and 27 using NIRC, the facility infrared camera (Matthews \& Soifer 1994). J-band images revealed that the brighter satellite was near its maximum separation on these nights, with an average distance of 1".4 from the primary. The average seeing was 0.5 on both nights. The signal from the satellite was easily separated from the primary in the raw imaging and spectral data. Figure 1 shows the $J$ band image of $2003 \mathrm{EL}_{61}$ and the outer satellite. The dimmer satellite was not detected.

Spectra of $2003 \mathrm{EL}_{61}$ and the brighter satellite were taken simultaneously by placing them in a $0.52 \times 38^{\prime \prime}$ slit. We obtained a spectrum in first order from 1.0 to $1.5 \mu \mathrm{m}$ by placing a 150 line $\mathrm{mm}^{-1}$ grism and a $J$ through $H$ sorting filter in the light path. A $1.4-2.5 \mu \mathrm{m}$ spectrum was obtained using a 150 line $\mathrm{mm}^{-1}$ grism and an $H$ through $K$ sorting filter. In $J-H$ our spectral resolution is approximately $\lambda / \Delta \lambda \approx 132$, and in $H-K$ the resolution is $\approx 162$. The spectra were acquired by taking $200 \mathrm{~s}$ exposures at five set dithered locations with a $5^{\prime \prime}$ separation between offset positions. The telescope guided at the predicted rate of motion for $2003 \mathrm{EL}_{61}$. The total integration time over both nights was $8000 \mathrm{~s}$ in $H-K$ and $2000 \mathrm{~s}$ in $J-H$. Nearby calibration stars were also observed each night at a range of air masses similar to those of $2003 \mathrm{EL}_{61}$. 

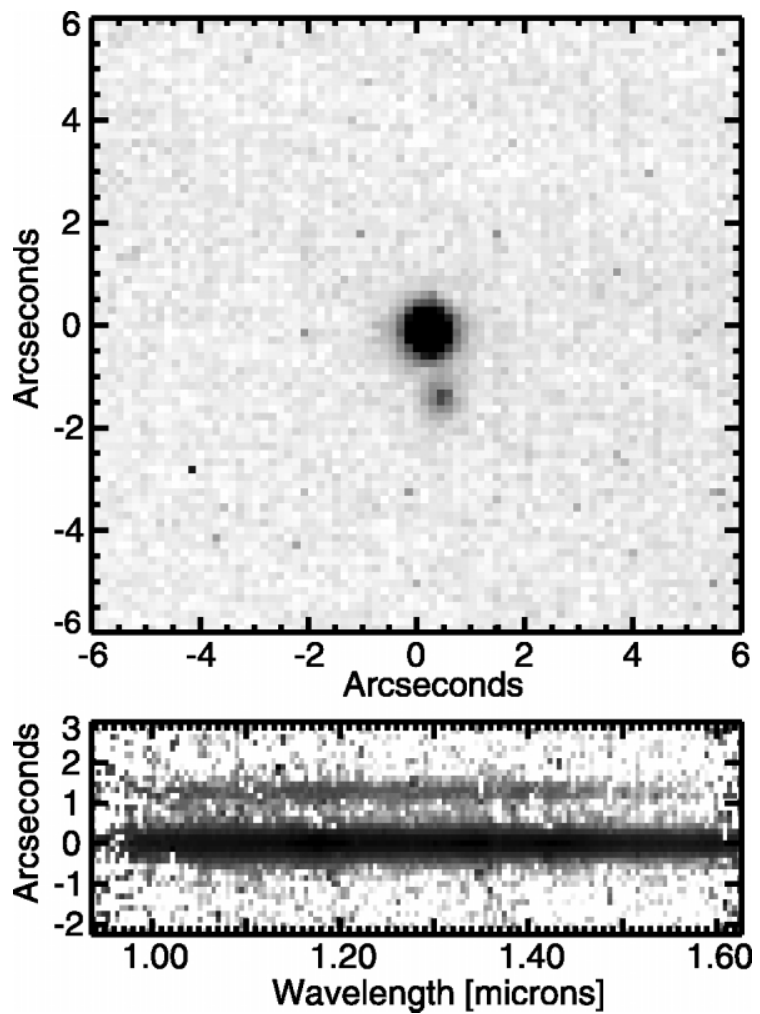

FIG. 1.- J-band image and raw spectral data of $2003 \mathrm{EL}_{61}$ and its outer satellite from UT 2005 April 27. The satellite was near its maximum separation of 1".4. The spectral data show that the satellite's spectrum is separated from $2003 \mathrm{EL}_{61}$.

\section{DATA REDUCTION}

The satellite's spectrum was marginally detected in the $200 \mathrm{~s}$ exposures. We hand-selected data in which the satellite spectrum was most clearly separated from the primary in the raw data for further analysis. In the $H-K$ band, a total of eight spectra from the first night and 10 from the second night were selected, reducing the total integration time to $3600 \mathrm{~s}$. In the $J-H$ band, three spectra from first night and four from the second were used, for a total integration $1400 \mathrm{~s}$. Data reduction was performed on the individual spectra in a manner similar to the procedures described in Brown (2000). After dividing each spectrum by a flat field, a majority of the sky background was removed by differencing adjacent pairs of spectra. The residual sky background was measured in 20 pixel-wide swaths above and below both $2003 \mathrm{EL}_{61}$ and the satellite. The average of the residual sky backgrounds was subtracted from every row of the spectrum. The spectra were then shifted to place $2003 \mathrm{EL}_{61}$ in the center of the array, and the individual spectra at similar air mass were added together to increase the signal from the satellite.

$2003 \mathrm{EL}_{61}$ contributes a few percent of the flux detected at the location of the satellite. To remove this contamination, a median profile of $2003 \mathrm{EL}_{61}$ was created in the spatial direction for each night. The profile of the data was then reflected across the point of maximum flux. At each column, the profile was then scaled by the flux from $2003 \mathrm{EL}_{61}$ in that column and subtracted from the co-added data. The spectrum of the satellite was extracted by summing over five rows centered at the location of the satellite. The final spectra were then divided by a calibration star spectrum to remove the solar spectrum, and the resulting reflectance spectra were averaged. The spectra were smoothed to increase the signal-to-noise ratio. The spec-

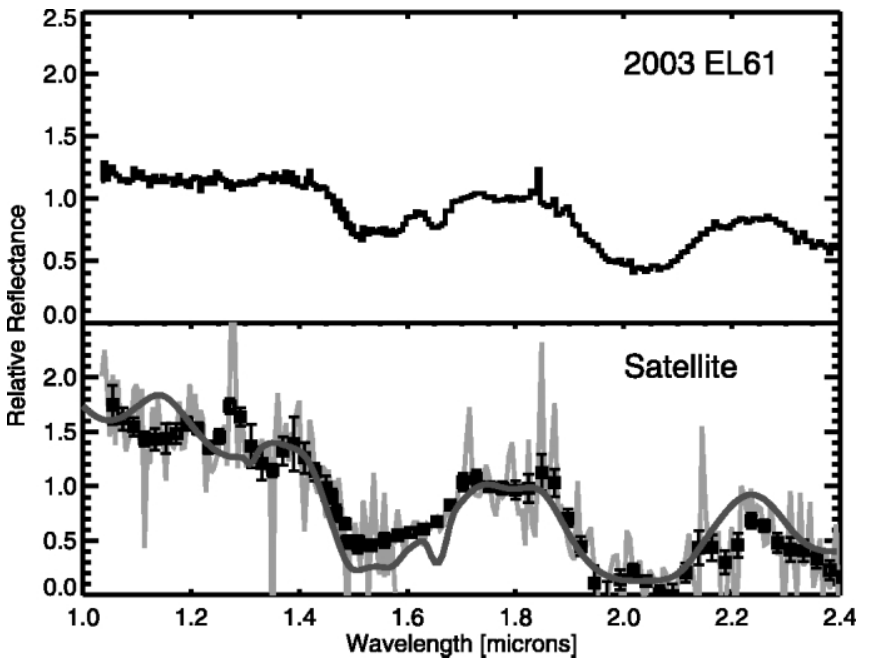

FIG. 2.-The 1.0-2.4 $\mu \mathrm{m}$ spectrum of $2003 \mathrm{EL}_{61}$ (top) and its outer satellite (bottom). Both spectra are scaled to the flux at $1.7 \mu \mathrm{m}$. The light gray line is the full-resolution spectrum of the satellite, and the squares are the Gaussiansmoothed data with error bars. The dark gray line shows our best-fit water ice model with a grain size of $250 \mu \mathrm{m}$. The resolution is insufficient to detect the $1.65 \mu \mathrm{m}$ crystalline water ice feature on the satellite. A slight blue component improves the model fit, but there is no evidence of other common ice species.

tral data were averaged at every fourth pixel using a Gaussian weighting function with an FWHM of 8 pixels in both the $J-H$ and $H-K$ data, resulting in an oversampled spectrum with a resolution of $\lambda / \Delta \lambda \approx 33$ in the $J-H$ data and 41 in the $H-K$ data.

For comparison, the spectrum of $2003 \mathrm{EL}_{61}$ was extracted in an identical manner to that of the outer satellite. The $H-$ $K$ data for both objects were normalized by the flux at $1.7 \mu \mathrm{m}$. The $J-H$ band overlaps with the $H-K$ band, and a scaling factor for the $J-H$ data was determined by fitting $2003 \mathrm{EL}_{61}$ 's $J-H$ and $H-K$ data, which have higher signal-to-noise ratios. This scaling factor was then used to scale the $J-H$ spectrum for the satellite.

\section{DISCUSSION}

The satellite spectrum shows strong absorption features at 1.5 and $2 \mu \mathrm{m}$, which are consistent with absorption due to water ice (Fig. 2). However, the resolution is too poor to detect the $1.65 \mu \mathrm{m}$ crystalline water ice absorption feature seen on $2003 \mathrm{EL}_{61}$ (Trujillo et al. 2005). The depth of the water lines suggests that much of the satellite's surface is covered with smooth water ice. We calculated a model water ice spectrum using laboratory optical constants from Grundy \& Schmitt (1998), assuming a temperature of 50 K. Our model uses Hapke theory (Hapke 1981) to transform the optical constants to a reflectance spectrum. We vary the grain size of ice in our models and find that the grain size of $250 \mu \mathrm{m}$ provides a good fit to the absorption lines at 1.5 and $2 \mu \mathrm{m}$. A better fit may be achieved with the addition of a second blue component or a multiple grain size water ice model. The poor fit of the water ice model shortward of $1.2 \mu \mathrm{m}$ is likely due to the uncertainties in the model in this region (Grundy \& Schmitt 1998). A deviation is also seen at $2.2 \mu \mathrm{m}$, where spectral features are sometimes reported (Cruikshank et al. 2005). However, the signalto-noise ratio of the spectrum is insufficient to identify an additional component with confidence.

The absorption features in the spectrum of the satellite are significantly deeper than water ice features typically observed 
on KBOs. The ratio of flux at $1.7 \mu \mathrm{m}$ to the flux at $2.0 \mu \mathrm{m}$ is $\sim 80 \%$. Most KBOs with water ice features have depths of less than $\sim 20 \%$, and the deepest absorptions $(\sim 60 \%)$ are found on $2003 \mathrm{EL}_{61}$, Charon, and $1996 \mathrm{TO}_{66}$ (see Brown et al. 2000, Fornasier et al. 2004, Dotto et al. 2003, and references therein). As such, the brighter satellite of $2003 \mathrm{EL}_{61}$ appears to be an unusual object in the Kuiper Belt. Captured objects are likely to resemble the parent population where they are formed; thus, the satellite's spectrum suggests that formation through capture is unlikely.

An alternative to capture is that the satellite could have formed via impact. Models of the Charon-forming impact by Canup (2005) are consistent with many of the features of the $2003 \mathrm{EL}_{61}$ system. In these models, Charon is likely formed from a grazing impact where the impactor remains mostly intact and creates a large satellite. However, the models also show that a more direct impact creates a disk around the target body and that smaller satellites can eventually coalesce from the disk material. The small satellites around $2003 \mathrm{EL}_{61}$ are more likely to be formed by the latter kind of impact than by the Charontype grazing impacts. In models where a disk was created, the satellite preferentially forms from the less dense disk material. In the outer solar system, this trend will lead to water-enriched satellites around KBOs, which may account for the strong water ice features seen in the satellite's spectrum. Direct impacts can also spin up the target body, which is consistent with the high rotation rate observed for $2003 \mathrm{EL}_{61}$ (Rabinowitz et al. 2006). $2003 \mathrm{EL}_{61}$ has a mean density of $2.6-3.4 \mathrm{~g} \mathrm{~cm}^{-3}$, indicating that it has lost most of its volatiles (Rabinowitz et al. 2006). Further modeling is needed to show whether or not a highenergy impact can eject a substantial amount of ice off of $2003 \mathrm{EL}_{61}$ to account for its high rock-to-ice ratio. In sum, $2003 \mathrm{EL}_{61}$ and its brighter satellite exhibit many of the features predicted by Kuiper Belt impact models. Further analysis of other satellites may provide us with a greater understanding of the dynamical history of the early Kuiper Belt.

The authors thank Chad Trujillo, David Rabinowitz, and Robin Canup for their helpful discussions regarding this Letter. We also thank Antonin Bouchez for his help during observations and our anonymous reviewer for the constructive comments. This research is funded by the California Institute of Technology and is also supported by the NASA Planetary Astronomy program. Data presented herein were obtained at the W. M. Keck Observatory, which is operated as a scientific partnership among the California Institute of Technology, the University of California, and the National Aeronautics and Space Administration. The observatory was made possible by the generous financial support of the W. M. Keck Foundation.

\section{REFERENCES}

Brown, M. E. 2000, AJ, 119, 977

Brown, M. E., Blake, G. A., \& Kessler, J. E. 2000, ApJ, 543, L163

Brown, M. E., \& Calvin, W. M. 2000, Science, 287, 107

Brown, M. E., et al. 2005, ApJ, 632, L45

- 2006, ApJ, 639, L43

Canup, R. M. 2005, Science, 307, 546

Cruikshank, D. P., Pilcher, C. B., \& Morrison, D. 1976, Science, 194, 835

Cruikshank, D. P., et al. 2005, Icarus, 175, 268

Dotto, E., Barucci, M. A., Boehnhardt, H., Romon, J., Doressoundiram, A., Peixinho, N., de Bergh, C., \& Lazzarin, M. 2003, Icarus, 162, 408

Fornasier, S., Dotto, E., Barucci, M. A., \& Barbieri, C. 2004, A\&A, 422, L43

Goldreich, P., Lithwick, Y., \& Sari, R. 2002, Nature, 420, 643
Grundy, W. M., \& Schmitt, B. 1998, J. Geophys. Res., 103, 25809

Hapke, B. 1981, J. Geophys. Res., 86, 3039

Licandro, J., Pinilla-Alonso, N., Pedani, M., Oliva, E., Tozzi, G. P., \& Grundy, W. M. 2006, A\&A, 445, L35

Matthews, K., \& Soifer, B. T. 1994, Exp. Astron., 3, 77

McKinnon, W. B. 1989, ApJ, 346, L109

Rabinowitz, D. L., Barkume, K., Brown, M. E., Roe, H., Schwartz, M., Tourtellotte, S., \& Trujillo, C. 2006, ApJ, 639, 1238

Stephens, D. C., \& Noll, K. S. 2006, AJ, 131, 1142

Trujillo, C. A., Barkume, K. M., Brown, M., Schaller, E. L., \& Rabinowitz, D. L. 2005, AAS/DPS Meeting Abstracts, 37, 52.06 